\title{
Le contrôle bancaire au regard des enjeux actuels des activités des banques privées en RDC - Entre la protection des clients, l'encouragement et la promotion des banques et la sécurité de l'Etat
}

\author{
Par Juslain NSAMBANA BONKAKO
}

\section{Résumé}

Le contrôle bancaire dans le système bancaire de la RD Congo est de deux types : le contrôle exercé par la Banque centrale du Congo et celui fait par les commissaires aux comptes. Cette étude s'est préoccupée à présenter l'intérêt de ce contrôle à l'égard des clients des banques, des pouvoirs publics comme pour les banques elles-mêmes. Il a été remarqué que le contrôle tel que réglementé et pratiqué ne permet pas d'assurer efficacement la protection des épargnants qui ne disposent pas d'action directe envers l'autorité de régulation et de contrôle (la banque centrale du Congo). De sorte qu'en cas des difficultés bancaires, les épargnants n'ont pas assez de garanties d'être payés. Nous avons ainsi suggérer, comme c'est le cas sous d'autres cieux, la création d'un Fonds de garantie de dépôts comme institution pouvant répondre des difficultés avérées d'une banque. L'intérêt pour l'Etat et l'humanité se justifie dans la nécessité de lutte contre le blanchiment des capitaux et le financement du terrorisme, dans la mesure où il s'est avéré que les activités des banques privées sont exposées à ces pratiques. Le contrôle bancaire constitue alors un moyen de prévention et de détection de ces fléaux. Il se fait malheureusement que les potentiels coupables de blanchiment des capitaux par le circuit bancaire sont des personnalités détentrices de pouvoirs censées assurer la répression.

\section{Summary}

Banking supervision in the banking system of the DR Congo is of two types: the control exercised by the Central Bank of Congo and that made by the auditors. This study is concerned to present the value of this control over the bank customers, public authorities and for the banks themselves. It was noted that control as regulated and practiced does not effectively protect the investors who have no direct action against the regulatory authority and control (the Central Bank of the Congo). So that in case of bank difficulties, investors do not have enough guarantees to be paid. We thus suggest, as is the case in other lands, the establishment of a deposit guarantee fund as an institution that can meet difficulties proved by a bank. The interest for the state and humanity is justified in the need to fight against

1 Assistant du professeur KUMBU ki NGIMBI, Faculté de Droit, Université de Kinshasa; Avocat et apprenant en DES 2015-2017; e-mail : jnsambana@gmail.com. 
money laundering and the financing of terrorism, insofar as it turned out that the activities of private banks are exposed to such practices. Banking supervision then is a means of prevention and detection of these scourges. It is unfortunately the money laundering potential culprits by the banking system are holders of power supposed to punish the offense.

\section{INTRODUCTION}

Dans tous les pays, l'activité bancaire est une profession réglementée. L'exercice des opérations de banques à titre de profession habituelle est soumis au respect des normes qui, non seulement, définissent les conditions à respecter mais aussi fixent les règles qui permettent d'assurer le contrôle desdites opérations. Ceci s'explique par le rôle aussi important que jouent les banques dans le monde, tous secteurs confondus.

Définie $^{2}$ comme tout établissement privé ou public qui facilite les paiements des particuliers et des entreprises, avance et reçoit des fonds, et gère des moyens de paiement, une banque privée remplit essentiellement trois missions pour intervenir tant dans la vie sociale des personnes que des affaires. Ces missions sont : - la réception et la collecte des fonds du public, - les opérations de crédit et celles de paiement et, - la gestion des moyens de paiement. Pour les remplir sans faille, elle a obligatoirement besoin d'importants revenus qui dépassent très souvent les capacités contributives de ses bailleurs ou actionnaires. Les dépôts qu'effectuent les clients par la suite sont un complément important qui permet à une banque privée de tenir tant soi peu ses activités. D'une part, d'importantes sommes mises à la disposition de banques sous forme de dépôts et, d'autre part, la place d'occupent les activités bancaires dans la vie des affaires nécessitent une surveillance toute particulière.

Le contrôle des banques dépend d'un pays à l'autre en fonction de son système bancaire. En RD Congo, en effet, la loi nº 003/2002 du 2 février 2002 relative à l'activité et au contrôle des établissements de crédit reconnait à la seule Banque Centrale du Congo (BCC) les prérogatives de contrôle des banques privées sur toute l'étendue de la RDC. C'est ainsi que dès la création d'une banque, il est préalablement requis l'agrément de la BCC. La Banque Centrale joue ainsi l'une de ses missions, de contrôle, en amont dans la mesure où toutes les banques créées passent nécessairement par cet agrément. Elle est, dans le secteur bancaire, l'œil et l'oreille de l'Etat en ce sens qu'elle contrôle les activités des banques privées et prend des mesures nécessaires pour la bonne marche dudit secteur. Dans ce dernier cas, elle édicte une réglementation pour réguler les activités des banques sur toute l'étendue de la RDC.

L'importance des activités des banques privées n'est plus à démontrer. Tous les secteurs de la vie trouvent, de quelque manière que ce soit, l'influence des activités des banques privées. Celles-ci, dans leurs missions, peuvent soit octroyer des crédits, dont l'objet, le béné-

2 Cette définition donnée par Le petit LAROUSSE, 2009, p 98, est reprise en des termes différents par d'autres auteurs dont Hervé ALEXANDRE (dir.), Banque et intermédiation financière,. 
ficiaire et l'échéancier les distinguent bien évidemment, soit faciliter des paiements des créances, soit enfin garder des fonds mis à leur disposition sous forme des dépôts.

Dans l'exercice de leurs activités, les banques privées gèrent à la fois les intérêts privés de leurs clients comme elles sont aussi soumises à certaines obligations légales et réglementaires leur imposées par l'Etat, à travers, dans le cas particulier de la RDC, la Banque Centrale du Congo. Dans telle ou telle autre hypothèse, des conflits sont évidents. Il est alors important que des mécanismes de contrôle des banques soient mis en place ou renforcés pour faire face à ces potentiels conflits.

En outre, l'évolution de certaines pratiques défendues à travers le monde suscite l'intérêt de renforcer le contrôle des banques privées dans la mesure où celles-ci jouent un certain rôle dans le financement ou la facilitation desdites pratiques. C'est le cas notamment du blanchiment des capitaux qui est devenu pratiquement un moyen par lequel d'importantes ressources de l'Etat sont détournés par des personnes qui en ont l'occasion. L'argent à origine délictueuse passe le plus souvent par le circuit des banques pour préparer son blanchiment. Dans ce contexte, le contrôle doit être exercé pour éviter les risques que ces activités présentent non seulement pour l'Etat mais aussi pour les clients et les banques elles-mêmes.

$\mathrm{Au}$ regard de ce qui précède, quelques questions sont fondamentales au sujet du contrôle bancaire au regard des enjeux actuels des activités des banques privées :

- Existe-t-il un cadre juridique bien fourni pour réglementer les activités des banques en RDC?

- Quelles sont ces activités des banques privées; quels en sont les risques éventuels à prévenir?

- Les mécanismes institutionnels de contrôle, s'il en existe, permettent-ils d'assurer véritablement le contrôle des banques privées et répondre à la triple préoccupation de garantir les intérêts de l'Etat, ceux des particuliers et la promotion de l'activité bancaire?

Pour répondre à ce questionnaire, quatre points seront examinés dans cette étude : une brève présentation du cadre juridique des activités des banques privées en RDC (A), le survol des activités des banques privées (B) et l'intérêt de renforcer le contrôle bancaire au regard des intérêts en compétition : clients, banques et pouvoirs publics (Etat) (C).

\section{A. Cadre juridique des activités des banques privées en RD Congo}

Par cadre juridique, il faut entendre l'ensemble des dispositifs normatifs mis en place pour organiser ou réguler les activités. C'est en d'autres termes tout l'arsenal juridique qui comprend, sur le plan national, la constitution, les lois, les ordonnances-loi, les ordonnances, les décrets, les arrêtés (ministériels ou des gouverneurs), les décisions, les circulaires, les instructions, etc. pour réglementer un secteur ou une activité donnée.

Le cadre juridique des activités des banques privées en RD Congo comprend non seulement des textes législatifs mais aussi une réglementation mise en place par la Banque Centrale du Congo sous forme de décisions, circulaires ou instructions. 


\section{Analyse de la législation congolaise sur les activités des banques privées}

En RD Congo, le secteur bancaire est actionné par plusieurs établissements de crédit, placés sous la supervision de la Banque centrale du Congo. Ces établissements de crédit, dont le nombre est de plus ou moins $167^{3}$, sont regroupés en cinq catégories distinctes, à savoir : les banques ${ }^{4}$, les coopératives d'épargne et de crédit, les caisses d'épargne, les institutions financières spécialisées et les sociétés financières.

En ce qui concerne uniquement les banques, pour rester dans le cadre restreint de la présente étude, trois lois seront succinctement examinés, sans préjudice toutefois des autres textes à porter général qui prévoient, à quelque manière que ce soit, certaines dispositions en rapport avec les banques. Les trois lois spécifiques sont : - la loi $\mathrm{n}^{\circ}$ 005-2002 du 7 mai 2002 relative à la constitution, à l'organisation et au fonctionnement de la Banque Centrale du Congo; - la loi n 003-2002 du 02 février 2002 relative à l'activité et au contrôle des établissements de crédit; et - la loi n ${ }^{\circ}$ 022-2002 du 30 octobre 2002 portant régime spécial de restructuration des établissements de crédit. Il y a lieu de noter que l'analyse de ces textes ne sera réservée qu'à leurs aspects en rapport avec le contrôle des banques qui constitue le cadre circonscrit de la présente étude.

\section{Le contrôle des banques privées en RDC}

En RDC, la base juridique du contrôle des banques privées est l'article 6 de la loi $\mathrm{n}^{\circ}$ 005-2002, précitée, qui dispose : " sans préjudice de l'objectif de stabilité du niveau général des prix énoncés à l'article 3, la Banque accomplit toutes missions de la Banque centrale, notamment : ... élaborer la réglementation et contrôler les établissements de crédit, les institutions de micro-finance et les autres intermédiaires financiers... ».

Prise trois mois après la loi $n^{\circ} 003 / 2002$, précitée, cette loi n ${ }^{\circ} 005-2002$ devait nécessairement prévoir, parmi les missions de la $\mathrm{BCC}$, l'élaboration de la réglementation et le contrôle des établissements de crédit pour assurer l'orthodoxie des textes et, surtout, insister sur le rôle de la BCC dans le système bancaire congolais. Cette loi n'a, par ailleurs, pas prévu de façon détaillée de règles devant organiser ledit contrôle. Il y a lieu donc d'interroger la loi $n^{\circ} 003 / 2002$.

En effet, la loi n003/2002 (appelée loi bancaire) édicte les règles que doivent respecter les établissements de crédit et celles auxquelles elles doivent se conformer. Cette loi confère ainsi à la $\mathrm{BCC}$ le pouvoir de superviser les établissements de crédit exerçant leurs

3 BCC, Rapport annuel 2013, Kinshasa, pp 197-301.

4 Dans son rapport annuel 2013, la banque centrale comptait 18 banques commerciales. 
activités en RDC. Elle définit ainsi des conditions d'ordre juridique ${ }^{5}$ et économique ${ }^{6}$ que doivent respecter les établissements de crédit. Ces conditions sont à observer tant lors de la constitution (création) qu'en cours d'activités de sorte que le contrôle exercé par la BCC consiste notamment en la vérification du respect de ces conditions.

Cette loi, contenant onze titres, prévoit concernant le contrôle bancaire deux organes compétents, à savoir la Banque Centrale du Congo et les commissaires aux comptes.

\section{a) Le contrôle effectué par la Banque Centrale du Congo}

L'article 36 de la loi $n^{\circ} 003 / 2002$, sous analyse, dispose que la banque centrale est chargée notamment de:

- Délivrer l'agrément des établissements de crédit, de leurs dirigeants et commissaires aux comptes ainsi que les autorisations ou dérogations individuelles, dans les limites fixées par les dispositions légales et réglementaires applicables aux établissements de crédit;

- Edicter la réglementation applicable aux établissements de crédit;

- Veiller au respect par les établissements de crédit, des dispositions légales et réglementaires qui leur sont applicables;

- Examiner les conditions d'exploitation des établissements de crédit;

- Veiller à la qualité de la situation financière des établissements de crédit et au respect de bonne conduite de la profession;

- Sanctionner les manquements aux dispositions légales et réglementaires applicables aux établissements de crédit.

Pour effectuer le contrôle des banques, deux types de contrôle sont prévus : sur pièces et sur place. Le premier consiste pour la Banque centrale d'apprécier la situation financière des établissements de crédit sur base des documents qui lui sont transmis par ces derniers. Le

5 Parmi les conditions d'ordre juridique, il y a lieu de noter que :

Les établissements de crédit sont constitués sous forme d'une personne morale dont, pour les banques spécialement la forme de société anonyme est obligatoirement requise;

Les dirigeants des établissements de crédit ne doivent pas être frappés d'interdiction professionnelle;

Ils doivent justifier d'un capital minimum, actuellement fixé à dix millions de dollar américain (10.000.000USD).

6 Les conditions d'ordre économique sont :

- La Banque Centrale du Congo vérifie si l'implantation de l'établissement de crédit répond à un besoin économique évident;

- Elle s'assure de la sécurité de la clientèle en contrôlant l'adéquation des moyens techniques et financiers de l'établissement de crédit à son programme d'activité;

- Lorsqu'il s'agit d'une filiale de banque étrangère, la Banque Centrale du Congo consulte les autorités de supervision du pays d'origine de cet établissement de crédit en vue de s'assurer de la crédibilité des promoteurs pour éviter l'introduction dans le circuit financier des capitaux d'origine criminelle. 
second, comme les termes l'indiquent, est celui par lequel la Banque centrale envoie ses inspecteurs sur terrain, au sein des établissements de crédit (les banques notamment) en vue de procéder à la vérification de l'exactitude des déclarations ou informations lui fournies.

Concernant le contrôle sur place, les établissements de crédit sont tenus de soumettre leurs encaisses, titres et valeur en portefeuille, ainsi que leurs livres, procès-verbaux, comptes, reçus et autres documents au contrôle de toute personne mandatée à cet effet par la Banque centrale, et de fournir à toute personne qui procède à ce contrôle toutes les informations et explications qui lui paraissent nécessaires ${ }^{7}$.

Ce contrôle permet à la Banque centrale, par le biais de ses inspecteurs délégués, de procéder à la vérification interne afin de déceler éventuellement des erreurs ou des fraudes et d'apprécier la qualité des procédures de décision, des systèmes de gestion ainsi que des dispositifs de maitrise de risque.

A l'issu de ces contrôles, la BCC peut, selon la gravité des faits :

- Adresser à charge de l'établissement coupable, une mise en garde, après avoir mis ses dirigeants en demeure de présenter leurs explications;

- Lui adresser une injonction à l'effet notamment de prendre, dans un délai déterminé, toutes mesures correctives appropriées;

- Prendre toute mesure de sauvegarde jugée nécessaire, notamment la désignation, pour une durée n'excédant pas six mois, d'un représentant provisoire de la Banque centrale;

- Nommer un administrateur provisoire ou un gérant provisoire à la tête de l'établissement de crédit;

- Retirer l'agrément.

Tel que le souligne Taylor LUBANGA, la finalité du contrôle de la Banque centrale (...) est avant tout préventive ${ }^{8}$ (et ensuite répressive ${ }^{9}$ ). Il s'agit en réalité de prévenir les conséquences d'une gestion imprudente pouvant conduire à une défaillance au regard de l'activité de l'établissement et qui peut causer préjudice à des tiers et surtout de s'assurer que le fonctionnement d'un établissement de crédit et la politique de crédit et de collecte des ressources suivie par les dirigeants ne font pas courir de risques excessifs.

7 Article 38 de la loi n003-2002, précitée.

8 Taylor LUBANGA, Précis de droit financier et bancaire. A la recherche d'un cadre institutionnel et juridique d'une effective marchéisation financière en RDC, Kinshasa, 2015, p. 74.

9 Le contrôle bancaire a, certes, comme objectif de prévenir les abus, mais la répression en cas de violation des règles mises en place n'est pas à méconnaitre. La répression permet de rendre efficace le contrôle en qu'elle peut aller jusqu'à mettre hors scène, les établissements coupables. Le retrait d'agrément par exemple peut être assimilé à une radiation; ce qui n'est pas évident pour un établissement de crédit. La répression est donc un aspect important du contrôle. 
b) Le contrôle bancaire des commissaires aux comptes : son intérêt pour l'Etat et les particuliers

Le contrôle effectué par des commissaires aux comptes dans une société commerciale (bancaire notamment) peut évidemment être perçu comme préservant les intérêts des associés ou actionnaires de ladite société. Ceci est d'autant plus convainquant que de par leur désignation, les commissaires aux comptes sont des mandataires d'actionnaires désignés aux fins d'exercer le contrôle sur les organes de gestion de la société ${ }^{10}$. Ils sont issus des assemblées d'actionnaires. La réalité est cependant autre en ce qui concerne les commissaires aux comptes des sociétés bancaires.

En effet, dans les banques, l'assemblée générale des actionnaires, associés ou sociétaires de chaque établissement de crédit est tenue de désigner en qualité de commissaire aux comptes soit deux personnes physiques, soit une personne morale parmi celles agréées par la Banque centrale. C'est dire que les commissaires aux comptes au sein des banques sont uniquement ceux agréés par la Banque centrale. Il y a lieu alors de comprendre qu'aucun commissaire aux comptes n'est méconnu de la banque centrale. La même loi prévoit à cet effet les conditions d'agrément des commissaires aux comptes ${ }^{11}$.

Les commissaires aux comptes sont donc des personnes dignes de confiance par l'autorité bancaire (la BCC). Le contrôle qu'ils effectuent tient compte de deux protections, audelà de l'intérêt supérieur de la banque privée. Ils sont à la fois mandataires des actionnaires de la banque privée, pour lesquels les intérêts doivent être protégés, et agréés de la Banque centrale, dont la décision du retrait d'agrément menace toute éventuelle légèreté des commissaires aux comptes. Ne serait-ce par cet élément psychologique, l'on peut présumer du sérieux de contrôle effectué par les commissaires aux comptes.

Les commissaires aux comptes ont trois missions qu'ils remplissent au sein d'une entreprise. Il s'agit de la mission d'information, de certification d'éléments autres que les comptes et de surveillance permanente ${ }^{12}$.

Dans le cadre de leur mission de surveillance permanente, les commissaires aux comptes soumettent à l'assemblée générale des actionnaires un rapport sur les comptes annuels de l'établissement de crédit conformément aux normes professionnelles en la matière. Une copie de rapport est communiquée à la Banque centrale.

Confrontés aux risques de retrait d'agrément, les commissaires aux comptes ont tout intérêt de remplir leur mission de contrôle avec compétence et intégrité. Le rapport dont copie est communiquée à la banque centrale constitue pour celle-ci, un élément de comparaison avec le rapport de mission qu'effectuent les inspecteurs de banque.

10 Lire pour plus des détails sur leurs désignation, missions et rémunérations, LUKOMBE NGHEN$D A$, Droit des sociétés, Kinshasa; BORIS MARTOR et alli, Le droit uniforme africain des affaires issu de l'OHADA, Paris, 2009.

11 C'est l'article 49, alinéa 2 de la loi n003-2002 qui prévoit ces conditions en fonction de la nature des commissaires aux comptes qui peuvent être des personnes physiques ou morales.

12 RAFFEGEAU et DUFILS, Audit et contrôle des comptes, Paris, sans date, p.22. 
En sa qualité d'autorité bancaire ${ }^{13}$, la banque centrale du Congo assure l'application de la législation sur les banques en vue de réguler le secteur bancaire par la mise en place d'une série d'instruments composant ce qu'il convient d'appeler « la réglementation bancaire au Congo ».

2. Analyse critique du système de contrôle bancaire en RDC : regard sur la protection des clients

La protection des clients des banques est une problématique qui peut être à la base de conflits entre l'Etat (via son organe régulateur) et ces banques, surtout dans le contexte de la RDC où le secteur bancaire est extraverti. La protection des particuliers (et donc des clients des banques) constitue une des missions les plus déterminants pour tout Etat indépendant et responsable.

Plusieurs questions se posent autour de la protection des clients des banques; c'est notamment sur la garantie des dépôts, sur le respect par les banques de taux d'intérêt réglementaire, sur le respect des conditions posées en début de relations d'affaires (octroi de crédit ou ouverture de compte bancaire), etc.; toutes ces questions tendent à réduire, sinon supprimer, l'inégalité qui se remarque dans les rapports banquiers et clients ${ }^{14}$.

Sans autre importante solution à entrevoir entre temps, le contrôle par l'Etat, garant des intérêts des particuliers, apparait comme la meilleure garantie pour ces derniers. La question demeure celle de savoir si le système bancaire congolais permet de garantir les intérêts des particuliers - clients des banques.

La garantie de dépôts est une question qui n'a pas encore trouvé de réponse appropriée en RD Congo. L'article 30 de la loi bancaire ${ }^{15}$, qui prévoit certaines possibilités pour la Banque centrale de mettre en place certains mécanismes de protection des dépôts, n'est pas à l'abri des critiques ${ }^{16}$. Au-delà du renforcement des règles juridiques en la matière, le contrôle de la Banque centrale devait tenir compte des problèmes réels des clients des banques. Une autre faille du système bancaire de la RD Congo, c'est de ne pas prévoir un

13 Lire $L U B A N G A$, note 8, p. 73.

14 Manuel Roland TCHEUMALIEU FANSI soutient avec raison, l'avons-nous estimé dans une étude antérieure, que les rapports banquier et client sont caractérisés par une très grande inégalité. Cette inégalité est très criante lorsqu'il s'agit du simple client particulier. Heureusement, le but de la réglementation est aussi de rétablir l'équilibre pour rassurer davantage les clients. (Le lire dans son ouvrage sur Le droit et pratique bancaire dans l'espace OHADA, Paris, 2013, p. 118). Nous l'avons en effet soutenu en partant simplement des conditions du contrat lors de l'ouverture d'un compte, préalable aux dépôts bancaires. Ces conditions sont arrêtées et modifiées par la banque sans moindre possibilité pour le client de participer aux échanges. Il ne fait que subir de sorte que tel contrat d'adhésion devient parfois léonin.

15 Loi n 003/2002, précitée.

16 Lire ces critiques dans Juslain NSAMBANA BONKAKO, « Le système bancaire de la République démocratique du Congo : analyse du cadre juridique et des mécanismes de contrôle pour protéger les clients ", in Librairie africaine d'études juridiques, disponible sur www.nomos-elibrairy.de. 
moyen de communication entre la Banque centrale, qui est sensée protéger, et les clients qui subissent les mauvaises pratiques des banques privées, qui sont sensés être protéger. Il n'est pas exclu que le contrôle effectué, en vue de la protection des clients, ne soit pas efficient. Sans plaider pour la mise en place d'une structure parallèle de contrôle, nous estimons que la banque centrale devait prévoir en son sein un mécanisme (division ou bureau) de représentation des clients des banques privées dont la mission essentielle consisterait en la gestion des plaintes des clients.

\section{La réglementation bancaire en $R D$ Congo : mission exclusive de la banque centrale du Congo}

Pour réglementer le secteur bancaire en RD Congo, la Banque centrale procède par des instructions et décisions. Si les instructions permettent d'organiser le secteur, de prévenir des abus et de déterminer les règles directrices à l'attention des établissements de crédit, les décisions interviennent pour réprimer les abus ${ }^{17}$.

Sans prétendre analyser dans le cadre de cette étude toutes les instructions prises par la Banque centrale, quelques unes concernant uniquement les banques seront examinées. C'est le cas notamment de l'instruction $\mathrm{n}^{\circ} 17$ aux établissements de crédit relative aux règles prudentielles en matière de contrôle interne et de conformité; et l'instruction $n^{\circ} 14$ aux banques relative aux normes prudentielles de gestion.

1. Instruction $n^{\circ} 14$ aux banques relative aux normes prudentielles de gestion

Cette instruction arrête les normes prudentielles applicables aux banques. L'article $1^{\text {er }}$ de cette instruction exige que les banques respectent de façon permanente les normes prudentielles de gestion fixées par la Banque Centrale du Congo.

Elle dresse en effet l'éventail desdites normes qui se rapportent essentiellement sur :

- Le capital minimum ${ }^{18}$

- Les fonds propres réglementaires

- La solvabilité

- La division des risques

- La surveillance des positions de change

- La liquidité

17 C'est le cas notamment d'une décision de la banque centrale qui retire l'agrément à un établissement de crédit, de celle qui prononce la dissolution et la liquidation d'un établissement de crédit, etc.

18 Actuellement, l'avons nous relevé, pour fonctionner, toute institution bancaire doit avoir un capital d'un montant minimum de dix millions de dollar américain logé à la BCC. Ce montant permet à l'autorité régulatrice du système bancaire de s'assurer de crédibilité économique de la banque à agréer. 
- La transformation à moyen terme et long terme

- La limitation des participations.

2. Instruction $\mathrm{n}^{\circ} 17$ aux établissements de crédit relative aux règles prudentielles en matière de contrôle interne et de conformité

Cette instruction rend obligatoire l'instauration du contrôle interne au sein des banques. Elle ne s'applique essentiellement qu'aux banques, institutions financières spécialisées, sociétés financières et caisses d'épargne.

Ce système de contrôle vise :

- A vérifier la régularité des opérations de l'organisation et des procédures internes

- A s'assurer de l'existence d'un suivi des risques et du respect des limites fixées par la banque centrale du Congo

- A veiller à la qualité des informations comptables et financières fournies et élaborées par les banques

Cette instruction a été mise en place en vue de permettre à la Banque centrale de faire un contrôle des banques grâce à des règles et méthodes comptables uniformes, ainsi que l'établissement des normes prudentielles sur base des données fiables.

\section{B. Les activités des banques privées en RD Congo : la réception et la gestion des fonds du public, l'accès au crédit et les risques de remboursement, le paiement des salaires par les banques, etc.}

Principalement ${ }^{19}$, toute banque remplit trois missions qui recadrent par conséquent ses activités, à savoir : la réception des fonds du public, l'octroi de crédits et les opérations de paiement et de gestion de moyens de paiement ${ }^{20}$.

\section{La réception et la garde des fonds du public : nécessité d'ouverture de compte bancaire}

De toutes les activités des banques privées, la réception des fonds du public est la plus voulue par ces dernières. Elle passe par l'ouverture de compte bancaire, lequel devra être alimenté par des dépôts de fonds. Il existe, en effet, plusieurs catégories de comptes qu'une personne peut ouvrir pour permettre à la banque de recevoir et garder son argent. Dans la pratique courante des banques commerciales en RD Congo, il existe principalement de compte courant et de compte épargne (appelé compte Ekonzo par la banque internationale

19 Certaines activités sont exceptionnelles au fonctionnement d'une banque. En RD Congo, c'est l'article 9 de la loi bancaire qui les énumère; c'est entre autres : les opérations de change, les opérations sur les métaux précieux, le placement, l'achat, la gestion, la garde et la vente de valeurs mobilières et tout produit financier, les prises de participation dans les limites fixées par la Banque centrale, etc.

$20 K U M B U$ ki NGIMBI, Législation en matière économique, Kinshasa, 2014, p. 87; TCHEUMALIEU, note 14, pp. 99-101; Taylor LUBANGA, note 8, p. 79. 
pour l'Afrique au Congo, Biac en sigle). La grande différence entre ces deux catégories des comptes fréquemment tenus par les banques privées réside dans leur fonctionnement, c'està-dire à la manière dont les mouvements de fonds (versements et retraits) sont gérés. Dans un compte courant, le client a en principe la possibilité tant d'effectuer des versements que de retirer en tout temps. Tandis que dans un compte épargne, tel que le mot l'indique, il s'agit d'un compte ouvert pour y effectuer des versements afin d'opérer le retrait à une échéance fixe. Acceptant d'ouvrir un compte épargne, le client consent implicitement à une interdiction de retirer à tout moment, malgré ses besoins avant l'échéance préalablement fixée.

Généralement, les taux d'intérêt diffèrent selon la nature de compte ouvert. Dans un compte courant, le taux est inférieur que celui appliqué dans un compte épargne.

Dans tous les cas, le compte de dépôt sert à enregistrer des encaissements et des paiements effectués par le banquier pour le compte de son client ${ }^{21}$. Par encaissement, il s'agit des versements personnels du client et des virements effectués pour son compte par une tierce personne; les paiements visent les retraits personnels du client ou les ordres de payer à une personne $\mathrm{X}$ ou encore l'exécution par la banque d'une injonction de payer issue d'une procédure de recouvrement à charge du client - titulaire du compte.

\section{L'octroi de crédit : quelle attitude pour les banques?}

Le crédit bancaire n'est rien d'autre que le prêt. De façon élémentaire, celui-ci désigne une opération par laquelle une personne, disposant de fonds, les « donnent » à une autre, qui les demande, à charge pour elle de rembourser à une échéance déterminée, avec intérêt. C'est cela qui permet à plusieurs auteurs de soutenir que l'opération de crédit est caractérisée par la réunion de deux éléments : le temps et l'argent ${ }^{22}$. En réalité l'octroi de crédit suppose que la banque a la liquidité qu'il faut pour servir celui qui demande.

L'octroi de crédit exige, de la part de la banque, la grande prudence en considération des risques inhérents à cette opération. C'est ainsi que à force d'être prudente, la banque est appelée à se poser des questions suivantes avant de prendre la décision d'octroyer un crédit à une personne :

- Combien faut-il prêter pour quelle catégorie de demandeur?

- Combien de temps faut-il donner pour recouvrer tel montant?

- Quelles sont des garanties à imposer pour s'assurer du recouvrement du montant prêté et ses intérêts?

- Quels sont des risques à encourir en prêtant telle somme à tel demandeur?

L'octroi précipité sans une étude responsable de ces questions expose la banque non seulement aux différentes crises (de liquidité par exemple) mais aussi aux conflits, parfois difficiles à résoudre.

21 LUBANGA, note 8, p. 55.

22 LUBANGA, note 8, p. 29. 
C'est dans ce contexte qu'il n'est pas étonnant qu'une banque effectue des missions d'évaluation des biens proposés pour garantir un crédit. L'évaluation permet donc à la banque de peser le risque ${ }^{23}$ qu'elle prend en octroyant le crédit; elle lui permet d'apprécier si la valeur du (des) bien(s) gagé(s) ou hypothéqué(s) est supérieure au crédit sollicité, au quel cas, la banque peut alors octroyer. Mais dans l'hypothèse inverse, c'est-à-dire que la valeur de biens mis en garantie est égale ou inférieure au crédit sollicité, la banque refuse carrément ou propose l'ajout des nouvelles garanties.

Les opérations de crédit présentent un intérêt majeur pour les entreprises, grandes, moyennes et petites, soient-elles.

\section{Les opérations de paiement : regard sur le paiement de salaire par voie bancaire en $R D C$}

Pour faciliter le paiement de créance entre opérateurs économiques, les banques interviennent comme intermédiaire financier. Le paiement par voie bancaire suppose que l'une des parties, ou toutes les deux, dispose d'un compte en banque qui lui permet soit de matérialiser l'opération. Selon le cas, il s'agira, pour le débiteur disposant d'un compte en banque d'émettre un chèque en faveur de son créancier ou, au cas où celui-ci est aussi titulaire d'un compte dans la même banque, d'ordonner à celle-ci d'opérer un virement pour le compte dudit créancier. Ceci entraîne donc un double mouvement de fonds : la banque débite le compte du débiteur pour créditer (alimenter) celui du créancier.

Dans des relations professionnelles entre travailleur et employeur, l'obligation de paiement de salaire constitue l'un des éléments caractéristiques du contrat de travail. La question qui s'ouvre à ce niveau est de savoir s'il est légal pour un employeur de procéder par une banque, dans le cadre de sa mission d'assurer les opérations de paiement, pour payer le salaire aux travailleurs ${ }^{24}$.

Le paiement de salaire par banque, appelé opération de bancarisation de paie, a gagné le terrain des employés du secteur public plutôt que privé en RDC.

23 Les banques encourent d'évidents risques en octroyant de crédits. Il est normal que les banques soient de plus en plus prudentes avant d'octroyer le crédit. L'octroi de crédit les expose notamment au non remboursement qui peut, même, entrainer leur faillite. Ces risques sont à lire avec plus de détails dans les publications de : Arnaud de SEVIGNY, et alli, Le risque de crédit, $3^{\text {ème }}$ éd, Paris, pp 12-13; Maria PSILLAKI, " contrats incitatifs et appréciation du risque », in Jacques SPINDLER (éd) : Contrôle des activités bancaires et risques financiers, Paris, 1998, p. 227; B. KEIZER, « La gestion des risques dans les banques ", in Revue d'économie financière, n 27, Hiver 1993; Dominique DUFOUR, «Réglementation prudentielle, économie du découvert et convention de financement, in jacques, SPINDLER (éd.) : contrôle des activités bancaires et risques financiers, Paris, 1998.

24 Théoriquement, dans le cadre de cette étude, nous envisageons élargir le sens des concepts « travailleurs » et « employeur ». En effet, par travailleurs, nous visons tous employés y compris les fonctionnaires et agents de l'Etat; l'employeur renvoie à tous ceux qui emploient lesdits travailleurs. 
Il semble que la bancarisation de paie des agents de l'Etat a permis de dégager l'effectif plus ou moins exact de ceux-ci. Conséquemment, le gouvernement de la RDC a pu rattraper une certaine somme d'argent ${ }^{25}$ dont les anciens bénéficiaires, qualifiés alors des fictifs, ne sont pas connus. Sur le plan de la gouvernance, il est donc avantageux d'envisager la couverture de tous les secteurs étatiques et paraétatiques.

Concernant le secteur privé, il y a lieu d'interroger la législation congolaise du travail pour voir si celle-ci pourrait être la cause de sous-bancarisation dans le secteur privé.

En effet l'article 98 du code du travail dispose : «La rémunération doit être payée en espèces, sous déduction éventuelle de la contre-valeur des avantages dus et remis en nature.

Le paiement doit avoir lieu pendant les heures de travail, au temps et au lieu convenus.

Le paiement de la rémunération ne peut avoir lieu dans un débit de boissons ni dans un magasin de vente, sauf pour les travailleurs employés dans ces établissements.

Il est interdit à l'employeur de restreindre de quelque manière que ce soit la liberté du travailleur de disposer de sa rémunération à son gré. ${ }^{26}{ }$

A la lumière de la disposition précitée, il y a lieu de dire que le législateur interdit tout autre paiement qu'en argent. En outre, le lieu et le temps peuvent être convenus entre employeur et travailleur. Ceci suppose qu'il n'est pas illégal de payer le salaire par la banque dans la mesure où celle-ci ne pourra finalement que payer en espèce; encore que la banque pourrait être prise comme lieu convenu. Ce qui est obligatoire, quant au temps en pareille hypothèse, est que l'employeur est tenu d'alimenter le compte du travailleur au temps convenu ou, à défaut de la convention, au plus tard dans les six jours qui suivent la période rémunérée ${ }^{27}$.

En définitive, dans tel ou tel autre secteur, ce qui doit être sauvegardé est que le paiement de la rémunération (d'un travailleur ou d'un agent de l'Etat) par voie bancaire n'occasionne pas de charges à supporter par le bénéficiaire. Ce dernier devrait être à mesure de toucher l'intégralité de sa rémunération; les charges occasionnées par les opérations bancaires, le coût de transport, spécialement pour ceux qui sont éloignés de leurs agences de perception, etc. ne devraient pas à notre sens être versés sur la tête du bénéficiaire.

$\mathrm{Au}$ vu de l'importance des activités des banques privées, il est absolument évident que le contrôle bancaire soit véritablement effectué pour protéger les intérêts en jeux.

25 Récemment la somme de 13 milliards de francs congolais a été rattrapée par le ministère congolais du budget.

26 Article 98 de la loi $n^{\circ} 015-2002$ du 16 octobre 2002 portant code du travail.

27 Article 99, al. 2 du code du travail. 


\section{L'efficacité du contrôle bancaire au regard des intérêts en compétition : banques, pouvoirs publics et clients des banques}

Le secteur bancaire est un des secteurs clés dans une économie nationale croissante quant à son enjeu dans la vie économique et sécuritaire. Il est donc du devoir du législateur d'ériger des remparts pour assurer le bon fonctionnement et garantir l'efficacité du système bancaire et mettre en place des mécanismes solides pour contrôler adéquatement le déroulement des opérations de banque. Le contrôle bancaire a pour but de réduire la probabilité de la faillite des banques, de veiller à la gestion prudente des fonds du public, de veiller à l'adéquation entre les crédits et les fonds propres des banques et de veiller à la transparence ainsi qu'à la conformité à la loi et aux règlements des opérations de banque, et enfin, de prévenir des éventuels dangers résultant des activités des banques.

La conduite des activités bancaires est essentiellement réservée aux seuls établissements de crédit agrées. Dès leur agrément, les établissements de crédit ainsi que leurs dirigeants sont assujettis à la réglementation qui organise l'activité bancaire. Cette réglementation organise non seulement les rapports qui doivent exister entre les établissements assujettis et les autorités compétentes mais également, elle organise la nature des rapports entre ces entités et leurs clients ${ }^{28}$.

C'est ainsi que le contrôle envisagé doit intéresser aussi bien les banques, leurs clients que les pouvoirs publics (l'Etat).

\section{Intérêt du contrôle bancaire pour les banques : le renforcement du contrôle est il favorable aux actionnaires de la banque privée et autres banques?}

Les banques privées, bien qu'évoluant dans le contexte du jeu concurrentiel, sont organisées et se rencontrent dans leurs activités. En effet, dans leur mission d'assurer les moyens de paiement, certains payements s'opèrent par virement entre banques. C'est dire qu'une banque privée est sensée être en harmonie avec d'autres banques. De même la faillite d'une banque, non seulement non voulue par ses actionnaires, n'est pas toujours accueillie par d'autres banques. Sachant que celle-ci est le plus souvent la cause de la mauvaise tenue des opérations et la gestion mal saine des risques qui entourent l'octroi de crédit, le contrôle se trouve être un moyen de remise sur les railles des organes de gestion courante d'une banque privée.

Pour les actionnaires d'une banque privée, le contrôle bancaire est un moyen qui leur permet de s'assurer de la bonne gestion de celle-ci. C'est à l'occasion du contrôle, effectué notamment par les commissaires aux comptes qu'ils ont mandatés, que les actionnaires arrivent, le cas échéant, à sanctionner les organes de gestion. Par le contrôle, les actionnaires peuvent se faire des idées sur le futur de la banque et prendre des résolutions pour éviter une éventuelle faillite bancaire dont les effets peuvent entamer leurs patrimoines indivi- 
duels en cas de déficit (c'est-à-dire que l'actif bancaire est inférieur aux obligations ${ }^{29}$ auxquelles la banque doit répondre).

Le contrôle présente également un avantage pour les autres banques en ce que dans le secteur, il existe des opérations interbancaires qui font que certaines banques puissent avoir des fonds dans d'autres. De même, l'activité bancaire est assise sur la confiance, pas envers une seule, mais plutôt à l'égard de tout le système, avec comme conséquence que toutes les banques sont concernées au cas où l'une d'elles est méfiée par les clients ${ }^{30}$.

Comme personne morale, une banque privée peut aussi être actionnaire d'une autre banque privée. Dans cette deuxième hypothèse, l'intérêt de renforcer le contrôle est évident dans la mesure où, comme actionnaire, les capitaux investis nécessitent d'être protégés.

\section{Intérêt du contrôle pour les clients des banques}

Dans une étude antérieure consacrée au système bancaire de la RD Congo : analyse du cadre juridique et des mécanismes de contrôle pour la protection des clients, nous avions dit que meilleure protection des clients de banques passe aussi en l'efficacité du contrôle bancaire.

En effet, rien n'est besoin de rappeler que les clients qui effectuent des dépôts en banque n'ont comme garantie que la crédibilité, je dirais mieux la confiance, vis-à-vis de la banque. Celle-ci pourtant, à l'hypothèse inverse d'octroi de crédit, est couverte d'importantes garanties pour prévenir des risques inhérents aux opérations de crédit.

Afin de protéger les intérêts des clients disposant des comptes en banques, la Banque centrale du Congo procède par le contrôle des normes prudentielles. Celles-ci sont l'ensemble des mesures de protection et de sécurité prises par la banque centrale pour assurer la protection des fonds du public déposés dans différents établissements de crédit.

Ces mesures de protection et de sécurité portent essentiellement sur :

- Le capital minimum dont doit disposer une banque;

- Les fonds propres d'une banque

- La solvabilité d'une banque

- La division des risques

- La surveillance des positions de change

- La liquidité

- La transformation à moyen et à long terme

- Limitation des participations

29 Par obligations, nous faisons allusion aux charges d'une banque privée qui regroupent : les impôts à payer au trésor public, les dettes obligataires, le traitement du personnel et, surtout, les dépôts des clients.

30 Lire également D. Ware, Basic Principes of Banking Supervision, cité par D. PUTU KIWANDA, Droit bancaire congolais, Kinshasa, 2015, p 234. 
Néanmoins, il n'est pas exclu qu'une banque privée, quelle que soit la qualité du contrôle, connaisse des difficultés de liquidité ou, carrément, de déséquilibre financier pouvant entrainer sa faillite. En pareille hypothèse, le déposant devrait être garanti du paiement de son avoir en banque. Ce qui justifie, au-delà du renforcement du contrôle (qui est une bonne attitude préventive dans la sauvegarde des intérêts des clients), la mise en place d'un véritable système de garantie de dépôts qui, en RDC et à l'exemple d'autres Etats du monde, devrait être à notre point de vu la création d'une institution appelée « Fonds de garantie de dépôt » ou « institution d'assurance dépôts bancaires ». Cette institution dont nous suggérons la nature privée, alimentée par des cotisations des banques privées au regard du volume des dépôts de chacune, aura pour mission de gérer des fonds en vue d'une intervention en faveur des déposants au cas où une banque en difficulté serait défaillante. Une loi devrait donc clairement préciser son organisation et son fonctionnement pour l'intérêt des clients.

III. Intérêt du contrôle bancaire pour les pouvoirs publics : la lutte contre le blanchiment des fonds

Les activités des banques privées en RDC comme à travers le monde sont exposées à plusieurs risques qui entament parfois gravement les intérêts de l'Etat. C'est le cas notamment du blanchiment des capitaux.

1. Lien entre les banques privées et la lutte contre le blanchiment de capitaux

Par définition ${ }^{31}$, le blanchiment est l'ensemble des opérations de nature économique ou financière dont l'objectif est l'insertion dans le circuit légal des capitaux ou des gains provenant d'activités illicites ou infractionnelles. Cette définition de la doctrine, plus explicite que celle légale, a l'avantage d'être comprise plus facilement.

L'article $1^{\text {er }}$ de la loi n ${ }^{\circ} 04 / 016$ du 19 juillet 2004 portant lutte contre le blanchiment des capitaux et le financement du terrorisme ${ }^{32}$ définit le blanchiment en référence aux actes constitutifs. En effet, dit l'article $1^{\mathrm{er}}$, au sens de la présente loi, sont considérés comme constitutifs de l'infraction de blanchiment de capitaux, les actes ci-dessous commis intentionnellement, à savoir :

$1^{\circ}$ la conversion, le transfert ou la manipulation des biens dans le but de dissimuler ou de déguiser l'origine illicite desdits biens ou d'aider toute personne qui est impliquée dans

31 Cette définition, adoptée par NYABIRUNGU mwene SONGA, est celle donnée par David HOTTE; lire NYABIRUNGU mwene SONGA, « le blanchiment des capitaux et le financement du terrorisme » in Annales de la Faculté de Droit, Université de Kinshasa, Kinshasa, juin 2014, p 407.

32 Journal officiel de la République Démocratique du Congo, $45^{\text {ème }}$ année, Numéro spécial, 5 août 2004. 
la commission de l'infraction principale à échapper aux conséquences juridiques de ses actes;

$2^{\circ}$ la dissimulation ou le déguisement de la nature, de l'origine, de l'emplacement, de la disposition, du mouvement ou de la propriété des biens;

$3^{\circ}$ l'acquisition, la détection ou l'utilisation des biens par une personne qui sait, qui suspecte ou qui aurait dû savoir que lesdits biens constituent un produit d'une infraction.

Ces actes constitutifs de blanchiment des capitaux passent généralement par le circuit bancaire pour être finalement servis au financement d'une activité ou d'un investissement qui sert de canal pour le blanchiment. C'est cela qui a suscité la motivation pour le législateur d'impliquer les banques dans la problématique de lutte contre le blanchiment des capitaux. Mais la question qui se pose est celle de savoir comment les banques peuvent concourir à cette lutte.

2. Rôle des banques dans la lutte contre le blanchiment des capitaux : analyse et critiques

L'activité bancaire est soumise à une série d'obligations contenues dans divers textes légaux et réglementaires. Parmi ces obligations, certaines sont liées aux intérêts de l'Etat et d'autres découlent plutôt de la protection des clients disposant des comptes en banques. Les premières visent notamment le concours des banques à la lutte contre le blanchiment des capitaux et le financement du terrorisme.

La loi sur la lutte contre le blanchiment des capitaux et le financement du terrorisme impose aux établissements de crédit (banques comprises) les obligations : de vigilance ${ }^{33}$, de rupture des relations d'affaires ${ }^{34}$ et d'information ${ }^{35}$.

Il est fait obligation aux établissements de crédit de s'assurer de l'identité aussi complète que possible du client avant d'établir toute relation d'affaires : ouvrir un compte ou livret, prendre en garde des titres, valeurs ou bons, attribuer un coffre ${ }^{36}$, etc.

Les clients des banques pouvant être des personnes morales, par identité complète pour cette catégorie des clients, l'on fait allusion à leur existence juridique réelle; c'est-à-dire que dans le dossier du client - personne morale, la banque doit avoir la certitude que la personne morale est régulièrement constituée en RDC ou à l'étranger. Les éléments tels que le numéro du registre de commerce et du crédit mobilier, l'identification nationale, le numéro d'impôt, le siège social d'une société commerciale - cliente de banque, sont obligatoires avant toute relation d'affaires.

Cette obligation de vigilance concerne aussi bien des clients habituels que des clients occasionnels. En plus du fait que dans tous les cas, l'identité du client doit rigoureusement être connue, concernant spécialement les clients occasionnels, lorsque l'opération effectuée

33 Articles 8 et 9 de la loi précitée sur le blanchiment des capitaux et le financement du terrorisme.

34 Article 10 de la même loi.

35 Article 11 de la même loi.

36 NYABIRUNGU mwene SONGA, note 30, p. 419. 
porte sur une somme égale ou supérieure à 10.000 dollars américains ou, bien qu'inférieur, soit qu'il y a doute sur la provenance licite des capitaux, soit encore qu'il y a eu, au cours des périodes rapprochées, répétition d'opérations, les banques sont tenues de renforcer leur vigilance par l'identification.

La rupture des relations d'affaires imposée aux banques dans le cadre de la lutte contre le blanchiment des capitaux est possible au cas où le doute persiste, quels que soient les efforts de la banque. La question qui se pose à ce propos est celle de savoir si la pratique dans des banques privées est en adéquation avec ce vœu du législateur.

Le blanchiment des capitaux en RDC est, comme on peut le présumer fermement en partant des constructions d'immeubles sur toute l'étendue de la république, fréquent dans des milieux des gouvernants plutôt que des simples opérateurs économiques. Il suffit de parcourir les différends chantiers de constructions d'immeubles et de sonder les voisins pour se rendre compte que notre affirmation n'est pas gratuite. La lutte contre le blanchiment dépasse la seule sphère bancaire car certains gros capitaux échappent au circuit des banques.

Dans le même ordre d'idée, le moyen de prévention de blanchiment, imaginée sur la vigilance des banques en cas d'opérations importantes, n'inquiète pas tellement le blanchisseur et ce, pour deux raisons :

- D'abord, celui-ci peut procéder par l'ouverture de plusieurs petits comptes en son nom ou aux noms des autres personnes qui lui sont proches pour s'échapper aux suspicions dues à l'importance du montant versé, s'ils tiennent à garder de l'argent à blanchir dans des banques;

- Ensuite, il peut carrément éviter les banques pour investir personnellement soit dans l'immobilier, soit dans d'autres activités lucratives dont le contrôle n'est pas très sévère. En définitive, comme le soutient le Professeur NYABIRUNGU ${ }^{37}$, le blanchiment des capitaux est un fléau pour le monde et pour nos Etats. La lutte engagée contre lui est nécessaire et de tous les instants pour la survie de l'humanité et de chacun de ces Etats car sa capacité de nuisance, de destruction voire d'anéantissement n'est plus à démontrer. La RD Congo, parmi tant d'autres pays qui visent l'émergence, a tout intérêt d'assurer efficacement le contrôle des banques en dépit de l'intérêt qu'elle a de les promouvoir. Un paradoxe peut alors être imaginé sur la double nécessité pour l'Etat de renforcer le contrôle des banques, ce qui pourrait devenir contraignant pour ces dernières, et aussi de promouvoir leurs activités au vu de leur importance dans le développement socio économique du pays.

\section{Conclusion}

La conduite des activités bancaires est essentiellement réservée aux seuls établissements de crédit agréés. Dès leur agrément, les établissements de crédit ainsi que leurs dirigeants sont 
assujettis à la réglementation qui organise l'activité bancaire ${ }^{38}$. Comprenant sur le plan interne deux catégories, cette réglementation fixe notamment des règles relatives au contrôle des banques privées dans l'exercice de leurs missions consistant principalement en la réception des fonds du public, en l'octroi de crédit, en des opérations de paiement et la gestion des moyens de paiement.

Le contrôle bancaire dans le système bancaire de la RD Congo est de deux types : le contrôle exercé par la Banque centrale du Congo et celui fait par les commissaires aux comptes. L'exercice du contrôle bancaire revêt un intérêt non seulement pour les banques elles-mêmes et leurs clients, mais également pour l'Etat et l'humanité. En effet, la protection des clients des banques ne peut être envisagée en dehors d'un véritable contrôle bancaire; ceci apparait, surtout dans le contexte de la RDC où les mécanismes de garantie de dépôts par exemple ne sont pas encore bien institués, comme le seul moyen qui permet de rassurer les clients. Devant ce souci de garantir les dépôts contre d'éventuelles difficultés que connaitraient les banques privées, la mise en place d'un Fonds d'assurances dépôts est une nécessité pour protéger les clients. Le Fonds ainsi créé devrait être une institution de gestion privée alimentée par les banques selon leur volume des dépôts. Cette institution privée devrait évidemment collaborer avec la banque centrale dans le cadre du contrôle bancaire.

L'intérêt pour l'Etat et l'humanité se justifie dans la nécessité de lutte contre le blanchiment des capitaux et le financement du terrorisme, dans la mesure où il s'est avéré que les activités des banques privées sont exposées à ces pratiques. Le contrôle bancaire constitue alors un moyen de prévention et de détection de ces fléaux; de même qu'il permet, le cas échéant, à l'Etat de réprimer certains cas repérés.

\section{REFERENCES BIBLIOGRAPHIQUES}

Arnaud de SEVIGNY, et alli, Le risque de crédit, $3^{\text {ème }}$ éd, Paris

BCC, Rapport annuel 2014, Kinshasa, 2015

BORIS MARTOR et alli, Le droit uniforme africain des affaires issu de l'OHADA, Paris, 2009.

DUFOUR Dominique, «Réglementation prudentielle, économie du découvert et convention de finan-

cement, in jacques, SPINDLER (éd.) : contrôle des activités bancaires et risques financiers, Paris, 1998.

KEIZER B., « La gestion des risques dans les banques », in Revue d'économie financière, $\mathrm{n}^{\circ} 27$, Hiver 1993

KUMBU ki NGIMBI, Législation en matière économique, Kinshasa, 2014

LUKOMBE NGHENDA, Droit des sociétés, Vol IV, Kinshasa,

Manuel Roland TCHEUMALIEU FANSI, Le droit et pratique bancaire dans l'espace OHADA, Paris, 2013. 
Maria PSILLAKI, " contrats incitatifs et appréciation du risque », in Jacques SPINDLER (éd) : Contrôle des activités bancaires et risques financiers, Paris, 1998,

NSAMBANA BONKAKO, «Le système bancaire de la République démocratique du Congo : analyse du cadre juridique et des mécanismes de contrôle pour protéger les clients ", in Librairie africaine d'études juridiques, disponible sur www.nomos-elibrairy.de

NYABIRUNGU mwene SONGA, « le blanchiment des capitaux et le financement du terrorisme » in Annales de la Faculté de Droit, Université de Kinshasa, Kinshasa, juin 2014

PUTU KIWANDA, Droit bancaire congolais, Kinshasa, 2015

RAFFEGEAU et DUFILS, Audit et contrôle des comptes, Paris, sd

Taylor LUBANGA, Précis de droit financier et bancaire. A la recherche d'un cadre institutionnel et juridique d'une effective marchéisation financière en RDC, Kinshasa, 2015 